\title{
SPHK1 promotes metastasis of thyroid carcinoma through activation of the S1P/S1PR3/Notch signaling pathway
}

\author{
ZHIJING ZHAO ${ }^{1}$, JUNFENG MA ${ }^{1}$, BAOQUAN HU $^{2}$, YI ZHANG ${ }^{2}$ and SHUSHU WANG $^{2}$ \\ ${ }^{1}$ Thyroid-Breast Surgery Department, The Second Affiliated Hospital of Kunming Medical University, \\ Kunming, Yunnan 650101; ${ }^{2}$ Breast Disease Center, Southwest Hospital, Third Military Medical University, \\ Chongqing 400038, P.R. China
}

Received August 6, 2017; Accepted December 21, 2017

DOI: $10.3892 /$ etm.2018.6054

\begin{abstract}
Thyroid carcinoma is characterized by an aggressive behavior, lack of effective targeted therapies and a high rate of relapse. Sphingosine kinase 1 (SPHK1) has been reported to be a critical regulatory factor in the progression of thyroid carcinoma, but the correlation between SPHK1 and clinical prognosis of patients with thyroid carcinoma has remained to be fully elucidated. The present study aimed to systematically assess the roles of SPHK1 in thyroid carcinoma metastasis and further investigate the possible underlying mechanisms. First, the expression of SPHK1 was detected in tissue samples from 53 thyroid carcinoma patients and in thyroid carcinoma cell lines by reverse transcription-quantitative polymerase chain reaction analysis. Furthermore, the level of phospho-(p)-SPHK1 was immunohistochemically detected in human thyroid carcinoma tissue samples. The activity of SPHK1 was measured with a commercial SPHK1 Activity Assay kit. A sphingosine-1-phosphate (S1P) competitive ELISA kit was used to determine the extracellular S1P levels. The metastatic potential was assessed by a Transwell assay. In addition, the association between SPHK1 and clinicopathological features of the patients was analyzed. The results indicated that the expression of SPHK1 in thyroid carcinoma samples was significantly higher than in paired adjacent normal thyroid tissues. High levels of SPHK1 were positively correlated with poor overall survival and progression-free survival. Downregulation of SPHK1 by lentiviral vector expressing SPHK1 small interfering (si)RNA evidently repressed Notch signaling and reduced the migration and invasion of thyroid carcinoma cells in vitro and in a NOD/SCID mouse model. Furthermore, inhibition of SPHK1 by siRNA or treatment with SPHK1 inhibitor 5C sensitized thyroid carcinoma to cisplatin and doxorubicin. In addition,
\end{abstract}

Correspondence to: Dr Shushu Wang, Breast Disease Center, Southwest Hospital, Third Military Medical University, 1 Main Street, Gaotanyan Road, Chongqing 400038, P.R. China

E-mail: wangshushu17@sina.com

Key words: thyroid carcinoma, sphingosine kinase 1, sphingosine1-phosphate, sphingosine-1-phosphate receptor 3, metastasis it was demonstrated that silencing of SPHK1 effectively inhibits processes associated with thyroid carcinoma metastasis through the Notch signaling pathway, and SPHK1 may therefore represent a potential therapeutic target in thyroid carcinoma. In conclusion, the present study indicated that high levels of p-SPHK1 were positively correlated with high levels of S1P which in turn promoted thyroid carcinoma metastasis via the S1P/S1P receptor 3/Notch signaling pathway, suggesting possible prognostic markers and therapeutic targets.

\section{Introduction}

Thyroid carcinoma (TC) is the most common type of malignant endocrine tumor and its incidence has increased rapidly in the past few decades $(1,2)$. When treating TC patients with standard therapies, including surgery, chemotherapy and radiotherapy, they are likely to acquire a good prognosis; however, the prognosis for patients with treatment resistance or tumor recurrence is poor (3). TC is divided into well-differentiated and undifferentiated types according to the histopathological and clinical characteristics. The well-differentiated types comprise papillary and follicular TC, and affected patients have a good prognosis. However, anaplastic TC, which are in the category of undifferentiated carcinoma, are aggressive and usually lethal, while they are less common (4-6). Although numerous studies have indicated that several important molecular markers are associated with TCs, which are accountable for the malignant transformation of thyroid cells, the biological behavior of TC cells, as well as their progression (7), the detailed mechanisms remain to be fully elucidated.

Sphingomyelin, cholesterol and other phospholipids are important components of the cell membrane, act as bioactive signaling molecules and have pivotal roles in TCs $(8,9)$. Among them, sphingosine-1-phosphate (S1P) is derived from sphingomyelin, and not only functions as an intracellular second messengers to regulate the cell cycle (10), but also binds to the specific receptors on the cell surface to regulate cell proliferation, apoptosis, invasion and migration $(5,10,11)$. In addition, S1P also promotes cell survival in multiple malignancies and simultaneously inhibits cell apoptosis, which is closely associated with tumor formation $(11,12)$.

Sphingosine kinase (SPHK) has a pivotal role in the process of S1P synthesis. SPHK occurs in two isoforms in humans and 
mice, SPHK1 and SPHK2. SPHK1 is predominantly located in the brain, heart, lung, liver, spleen and hematopoietic immune system, and is the most important molecule to regulate the generation of S1P $(9,13)$. Huang and Natarajan (14) reported that NIH 3T3 fibroblasts with overexpression of SPHK1 acquired transformed phenotypes and that tumorigenesis was promoted, which implied that SPHK1 has a marked oncogenic activity. Numerous studies have also demonstrated that the expression of SPHK1 was elevated in multiple human tumor types, and was extensively involved in head and neck squamous cell carcinoma (14), glioma (15), salivary gland carcinoma (16), prostate cancer (17), non-Hodgkin lymphomas (18), gastric cancer and papillary TC (14-19). Furthermore, upregulation of SPHK1 in certain cancer types is closely associated with a worse clinical prognosis (20). Brünnert et al (21) also indicated that upregulation of SPHK1 expression induced epithelial growth factor (EGF) receptor in gastric cancer via interaction with lysophosphatidic acid (LPA) receptor. Subsequently, other studies have reported that knockdown of SPHK1 inhibited the induction of EGF in MCF7 cells and decreased the migration of 293 cells induced by EGF (22). Taken together, these studies implied that SPHK1/S1P may be a key regulator of tumor invasion and metastasis. However, despite increasing evidence demonstrating that SPHK1 is elevated in TC, the therapeutic implications of SPHK1 and the associated molecular mechanisms have remained largely elusive.

The present study first determined the expression of SPHK1 in TC tissues and cell lines compared with that in paired normal thyroid tissues and a normal thyroid cell line. Furthermore, the effects of SPHK1 on the migration and invasion of TC cells were assessed in vitro, and the association between the expression of SPHK1 and the metastatic potential of TC cells was explored in vivo. Subsequently, the underlying molecular mechanisms were investigated. The present study provided an experimental basis for the utilization of SPHK1 as a potential therapeutic target in TC.

\section{Materials and methods}

Patients and samples. TC and adjacent normal tissues were collected from a total of 53 patients who were diagnosed with TC and underwent surgical resection at the Southwest Hospital of the Third Military Medical University (Chongqing, China) between December 2015 and December 2016. These patients had invasive tumors and the tumor diameter was $>2 \mathrm{~cm}$. Samples were collected to ensure the safety of patients. All collected tissue samples were snap-frozen and then stored at $-80^{\circ} \mathrm{C}$. All of the patients provided informed consent and the present study was approved by the medical ethics committee of the Third Military Medical University (Chongqing, China).

Cell lines and reagents. The human TC cell lines SW579, TPC-1 and WRO, and the normal thyroid cell line Nthy-ori 3-1 were obtained from the American Type Culture Collection (Manassas, VA, USA), cultured in RPMI-1640 medium (Gibco; Thermo Fisher Scientific, Inc., Waltham, MA, USA) supplemented with $10 \%$ heat-inactivated fetal bovine serum (FBS; Gibco; Thermo Fisher Scientific, Inc.) and 100X penicillin-streptomycin solution. The human K1 thyroid gland papillary carcinoma cell line is a misidentified cell line that has been reported to be a derivative of the GLAG-66 cell line of the same cancer type (23), and was cultured in Dulbecco's modified Eagle's medium (Gibco; Thermo Fisher Scientific, Inc.) supplemented with 10\% FBS and antibiotics. The normal thyroid cell line Nthy-ori 3-1 was used as a normal control. Cisplatin and doxorubicin were purchased from Sigma-Aldrich (Merck KGaA, Darmstadt, Germany) and dissolved in dimethyl sulfoxide. S1P was purchased from Enzo Life Sciences (Farmingdale, NY, USA). 5C, an inhibitor of SPHK1, was obtained from Santa Cruz Biotechnology, Inc., (Dallas, TX, USA). JTE013, CAY10444 and TY52156 were purchased from MedChem Express (Monmouth Junction, NJ, USA). LPA was obtained from Avanti Polar Lipids (Alabaster, AL, USA). Anti-hairy and enhancer of split-1 (Hes1) antibody (cat. no. ab49170) and anti-SPHK1 antibody (cat. no. ab71700) were obtained from Abcam (Cambridge, UK), while rabbit GAPDH monoclonal antibody (cat. no. 2118) was obtained from Cell Signaling Technology Inc. (Danvers, MA, USA). Anti-Phospho-SPHK1 antibodies (cat. no. PAB16439) were purchased from Bio-Swamp Life Science Lab (Wuhan, China). Notch1 intracellular domain (N1ICD) antibody was obtained from EMD Millipore (Billerica, MA, USA).

Lentiviral transfection. The TC cell lines TPC-1 and WRO were seeded into 6-well plates at a density of $2 \times 10^{5}$ cells/well. One day after adherence, cells were maintained in RPMI-1640 medium containing $2 \%$ FBS. The siRNA was synthesized by Beyotime Institute of Biotechnology (Haimen, China) with following sequences: Lv-NC-siRNA: 5'-UUCUCCGAACGU GUCACGUTT-3', Lv-SPHK1-siRNA\#1: Sense 5'-GCAGCU UCCUUGAACCAUUTT-3' and Lv-SPHK1-siRNA\#2: Sense 5'-GUGCACCCAAACUACUUCUTT-3'. Transfection of siRNA was performed with ViraPower ${ }^{\mathrm{TM}}$ (Invitrogen; Thermo Fisher Scientific, Inc.) following the manufacturer's instructions (14). These cells were transfected with Lv-NC-siRNA or Lv-SPHK1-siRNA at a multiplicity of infection of 20. After $10 \mathrm{~h}$ of incubation, the medium was changed. The transfection efficiency was determined as established by a previous study (14) and cells were used for further study.

Immunohistochemistry (IHC). All TC tissue and normal adjacent samples were submitted to the Department of Surgical Pathology of the Southwest Hospital of the Third Military Medical University (Chongqing, China) and examined by three experienced pathologists. Paraffin-embedded blocks from each selected specimen were used for IHC. Serial $4 \mathrm{~mm}$ sections were used for staining for SPHK1 $(1: 5,000)$ and p-SPHK1 $(1: 5,000)$. IHC were performed as previously described (24).

Animal study of in vivo metastasis formation. A total of 15 healthy 4-week-old NOD/SCID mice (male, $20 \pm 1 \mathrm{~g}$ ) were purchased from the Animal Center of the Third Military Medical University (Chongqing, China). These mice were collected and maintained in a pathogen-free environment at the animal facility of the Third Military Medical University (Chongqing, China) according to the Care and Use or Experimental Animals (Permission no. SYXK-PLA-20120031). All procedures for animal experiments were approved by the Laboratory Animal Welfare and 
Ethics Committee of the Third Military Medical University (Chongqing, China; no. 20151117083) and were performed in accordance with institutional guidelines. The mice were randomly divided into a control group (which received an equal quantity of normal saline to the siRNAs), a lentiviral vector expressing negative control (NC) small interfering (si)RNA (Lv-NC-siRNA) and an Lv-SPHK1-siRNA\#1 group. For the in vivo metastasis assay, $2 \times 10^{5} \mathrm{TPC}-1$ cells transfected with Lv-NC-siRNA or Lv-SPHK1-siRNA\#1 or control treatment were mixed with Matrigel (BD Biosciences, Franklin Lakes, NJ, USA) at a ratio of $1: 1$ and then injected into the spleen of the NOD/SCID mice (under $80 \mathrm{mg} / \mathrm{kg}$ pentobarbital IV. anesthesia and with the abdomen open according to general procedures) (18). After 6 weeks, all animals were sacrificed by carbon dioxide euthanasia (no more than $30 \%$ of the chamber volume/minute) and imaged using an in vivo Imaging System (Bio-Real in vivo imaging system; QuickView3000; Bio-Real Sciences; LABATECH GmbH; Salzburg, Austria) to examine nodule tumor metastasis in different organs and to assess the weight of tumors.

Measurement of SPHK1 activity. The activity of SPHK1 was measured with a commercial SPHK1 Activity Assay kit (cat. no. KA0906; Abnova, Taipei, Taiwan) according to the manufacturer's instructions.

Measurement of S1P. An S1P competitive ELISA kit (cat. no, K-1900, Echelon Bioscience, Inc., Salt Lake City, UT, USA) was used for detecting S1P levels. Cells were seeded onto 6-well plates at $10^{5}$ cells/well without FBS and allowed to attach for $12 \mathrm{~h}$. Subsequently, the cells were pre-treated with inhibitors for another $15 \mathrm{~min}$ prior to stimulation with $10 \%$ FBS for $6 \mathrm{~h}$. The supernatant was collected for S1P analysis according to manufacturer's instructions.

Cell viability assay. The Cell Counting Kit-8 (CCK-8) assay was used for determining the viability of TC cells. TPC-1 and WRO cells were seeded onto 96-well plates at a density of $1 \times 10^{4}$ cells/well (attached prior to the drug treatment) and then treated with cisplatin or doxorubicin at $10 \mu \mathrm{M}$ for $48 \mathrm{~h}$. CCK-8 solution was added into 96-well plates at the end of the experiment, followed by incubation for $3 \mathrm{~h}$ at $37^{\circ} \mathrm{C}$. The absorbance at $450 \mathrm{~nm}$ was read with a microplate reader (Bio-Rad Laboratories, Inc., Hercules, CA, USA) and the cell viability relative to the control group was calculated. The experiment was repeated 3 times following the first experiment.

Western blot analysis. Treated TPC-1 TC cells were collected and lysed with ultrasonication (VOSHIN96-II; Wuxi Voshin Instruments Co., Ltd., Wuxi, China) on ice for $30 \mathrm{~min}$ (rest for $5 \mathrm{sec}$ every $5 \mathrm{sec}$ ), followed by centrifugation of the lysate. The protein concentration in the supernatant was determined using a BCA assay. For each sample, $20 \mu \mathrm{g}$ protein was loaded, subjected to $6 \%$ SDS-PAGE and transferred onto PVDF membranes (cat. no. AR0136-04, Whuan Boster Biological Technology ltd., Wuhan, China). Membranes were blocked for $0.5 \mathrm{~h}$ at room temperature using TBST with $5 \%$ skimmed milk and then incubated with Hes1 $(1: 5,000)$, N1ICD $(1: 5,000)$ and GAPDH $(1: 10,000)$ antibody at $4^{\circ} \mathrm{C}$ for overnight.
Subsequently, the membranes were incubated with secondary antibody (horseradish peroxidase conjugated Anti-Mouse Immunoglobulin G H\&L; cat. no. ab6789, Abcam) for $2 \mathrm{~h}$ at room temperature. The signals were detected by enhanced chemiluminescence (Pierce; Thermo Fisher Scientific, Inc.). GAPDH was regarded as an internal control.

Reverse transcription-quantitative polymerase chain reaction (RT-qPCR) assay. Total RNA was isolated with TRIzol (cat no. 12183555, Invitrogen; Thermo Fisher Scientific, Inc.) from the tissues of TC patients and cell lines according to the manufacturer's protocols. The concentration and integrity total RNA was determined with a NanoDrop-1000 (Thermo Fisher Scientific, Inc.) and by electrophoresis, respectively. A reverse transcription kit (cat. no.: A5001, Promega Corp., Madison, WI, USA) was used for synthesis of the first-strand complementary (c)DNA. Real-time qPCR was used to amplify and quantify the cDNA of SPHK1 (forward primer, 5'-GGAGGA GGCAGAGATAAC-3'; reverse primer, 5'-TTAGCCCATTCA CCACTTCA-3'), dickkopf-1 (DKK1; forward primer, 5'-CCT TGGATGGGTATTCCAGA-3'; reverse primer, 5'-CCTGAG GCACAGTCTGATGA-3'), Hes1 (forward primer, 5'-ACC TTCCAGTGGCTCCTC-3'; reverse primer, 5'-TTTAGTGTC CGTCAGAAGAGAG-3') and Gli1 (forward primer, 5'-GCC ATGAAACTTTCACCGTG-3'; reverse primer, 5'-TCTGGG GGGTTACCAAGTTA-3') with ABI 7900HT Real-Time PCR System (Applied Biosystems, Carlsbad, CA, USA) using One Step PrimeScript ${ }^{\mathrm{TM}}$ RT-PCR kit (cat no. RR064A; Takara Biotechnology Co., Ltd., Dalian, China). GAPDH (forward primer, 5'-CGGAGTCAACGGATTTGGTCGTAT-3'; reverse primer, 5'-AGCCTTCTCCATGGTGGTGAAGAC-3') mRNA was considered as an internal control. The $\Delta \Delta \mathrm{Cq}$ method was applied for quantification following previous reports (25).

Migration and invasion assays. TC cells re-suspended in serum-free RPMI-1640 medium were seeded into the upper chambers of a Transwell insert $(8.0 \mu \mathrm{m}$, EMD Millipore) at $3 \times 10^{4}$ cells/well. RPMI-1640 medium containing 20\% FBS was added to the lower chamber of 24-well plates (Corning Inc., Corning, NY, USA). For the invasion assay, the upper side of the filter was covered with Matrigel (BD Biosciences) and $1 \times 10^{5}$ cells per well were seeded onto the upper chambers. After incubation for $24 \mathrm{~h}$, cells on the upper side of the membrane were removed and the filter membrane was fixed with $4 \%$ paraformaldehyde and stained with $0.5 \%$ crystal violet (Beyotime Institute of Biotechnology, Haimen, China). The numbers of cells were counted in ten fields for each sample.

Statistical analysis. Values are expressed as the mean \pm standard deviation. Statistical significance was analyzed by SPSS version 16.0 statistical software (SPSS, Inc., Chicago, IL, USA). A bivariate analysis was used to assess differences in multiple groups (Student's t-test, one-way analysis of variance with multiple comparisons by a post-hoc Tukey-honest significant difference test for independent samples). A Kaplan-Meyer survival analysis was also applied. Pearson's correlation analysis was selected to determine the correlation of SPHK1 expression and metastatic potential. $\mathrm{P}<0.05$ was considered to indicate a statistically significant difference. 
A

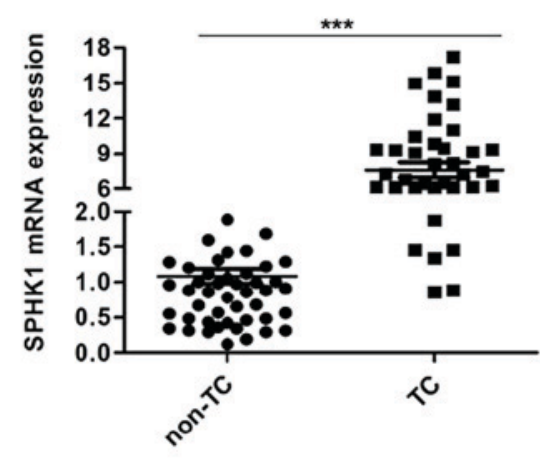

C

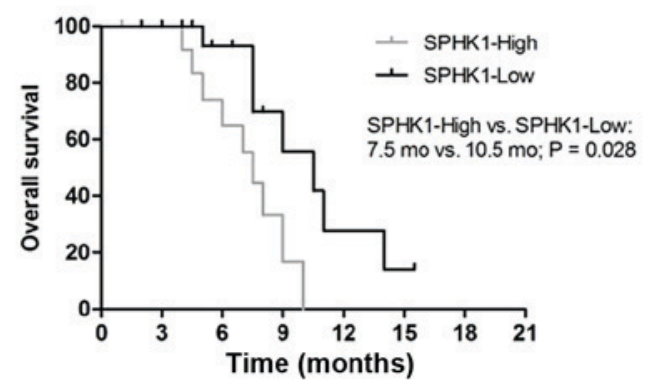

B

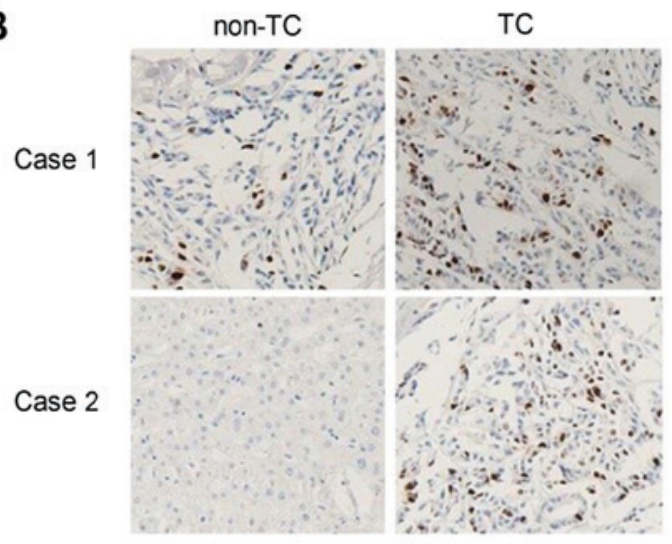

D

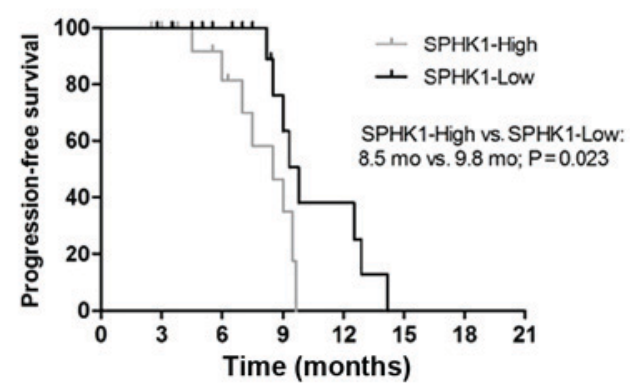

Figure 1. SPHK1 levels are upregulated in TC tissues. (A) SPHK1 expression was detected by reverse transcription-quantitative polymerase chain reaction analysis in TC tissues and paired adjacent normal thyroid tissues. Each data-point represents the value for one individual patient. (B) Representative immunohistochemical images for the detection of SPHK1 expression (brown stain) in TC and non-TC tissues (magnification, x40). (C and D) Kaplan-Meier plots for (C) overall survival and (D) progression-free survival of all TC patients. SPHK1, sphingosine kinase 1; TC, thyroid carcinoma. Values are expressed as the mean \pm standard deviation. ${ }^{* * *} \mathrm{P}<0.001$. SPHK1, sphingosine kinase 1 ; TC, thyroid carcinoma.
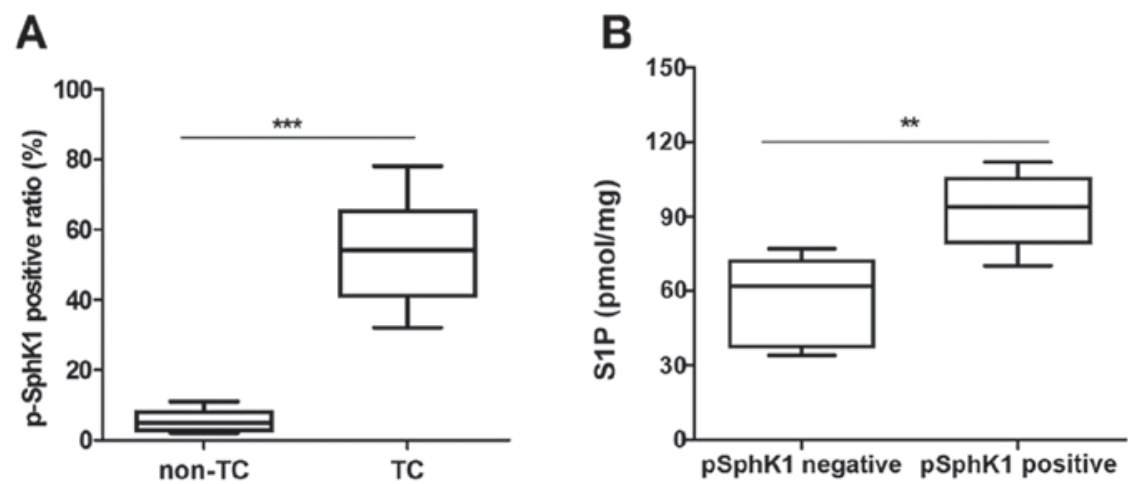

Figure 2. p-SPHK1 levels in TC patients are higher compared with those in adjacent normal thyroid tissues and the levels of S1P are correlated with high expression of p-SPHK1. (A) p-SPHK1 levels were detected in TC patients and adjacent normal thyroid tissues by immunohistochemistry and the p-SPHK1-positive ratio was calculated. (B) The levels of S1P were detected in TC patient cell supernatants as positive or negative for p-SPHK1. p-SPHK1, phosphorylated sphingosine kinase 1; TC, thyroid carcinoma; S1P, sphingosine-1-phosphate. Values are expressed as the mean \pm standard deviation. ${ }^{* * *} \mathrm{P}<0.01,{ }^{* * * *} \mathrm{P}<0.001$. SPHK1, sphingosine kinase 1; TC, thyroid carcinoma.

\section{Results}

SPHK1 is upregulated in human TC tissues. The expression of SPHK1 in human TC tissue samples and adjacent normal thyroid (non-TC) tissue samples from 53 patients was determined by RT-qPCR. The results indicated that the mRNA expression of SPHK1 was evidently elevated (at least 5-fold) in human TC tissue samples compared with that in non-TC tissue samples in 40 of 53 patients $(75.47 \%$; $\mathrm{P}<0.05$, Fig. 1A).
Furthermore, the protein levels of SPHK1 were detected in TC tissue samples and non-TC tissue samples by IHC. The protein levels of SPHK1 were obviously higher in TC samples compared with those in non-TC samples and representative images are presented in Fig. 1B. All TC patients were divided into an SPHK1-high and an SPHK1-low group according to the levels of SPHK1, and a Kaplan-Meier survival analysis indicated that the level of SPHK1 was negatively correlated with overall survival (OS; $\mathrm{P}=0.028$; Fig. $1 \mathrm{C}$ ) and progression-free 
survival (PFS; $\mathrm{P}=0.023$; Fig. 1D) of TC patients. Taken together, the results indicated that high levels of SPHK1 are correlated with a poorer survival and prognosis of TC patients.

Overexpression of SPHK1 in TC patients, as well as S1P levels, are positively correlated with high levels of phosphorylated ( $p)$-SPHK1. A previous study indicated that p-SPHK1 (Ser-225) is associated with S1P export (15). Thus, the present study further determined the levels of p-SPHK1 in human TC tissue samples and non-TC tissue samples by IHC. It was observed that TC tissue samples had higher p-SPHK1 levels compared with non-TC tissue samples (Fig. 2A). In addition, the level of S1P export in cell supernatants were evidently higher in p-SPHK1-positive tumors compared with that in p-SPHK1-negative tumors. These results demonstrated that S1P levels are obviously associated with p-SPHK1 levels in human TC patients (Fig. 2B). Furthermore, the association between p-SPHK1 levels and clinicopathological factors (TNM stage) in human TC patients is presented in Table I.

Overexpression of SPHK1 in human TC cells and their metastatic capacity is positively correlated with SPHK1 levels. To further investigate the roles of SPHK1 in TC progression, the expression of SPHK1 was detected in the human TC cell lines SW579, K1, TPC-1 and WRO, compared with that in the normal thyroid cell line Nthy-ori 3-1 as a control. The results indicated that the mRNA levels of SPHK1 were significantly higher (at least 2-fold) in the TC cell lines compared with those in the normal thyroid cell line Nthy-ori 3-1 (Fig. 3A). In addition, the activity of SPHK1 was measured using an SPHK1 activity assay kit, which indicated that SPHK1 activity was higher in TC cell lines than in Nthy-ori 3-1 (Fig. 3B). Furthermore, the TC cell lines TPC-1 and K1 exhibited a more pronounced migration ability than WRO, SW579 and Nthy-ori 3-1 (Fig. 3C). Further analysis suggested that the migration ability of TC cells was positively correlated with SPHK1 expression (Fig. 3D). Similar results were also obtained regarding the invasion ability of TC cells (Fig. 3E and F). Taken together, these results demonstrated that the ability of TC cells to form metastases was positively correlated with SPHK1 levels.

Silencing of SPHK1 expression impairs the migration and invasion ability of TC cells, and reduces metastasis of TPC-1 cells in NOD/SCID mice. To determine whether SPHK1 levels are associated with the ability of TC cells migrate and invade, a TPC-1 cell model was selected for further investigation. TPC-1 cells were transfected with lentivirus expressing SPHK1 siRNAs (Lv-SPHK1-siRNA\#1 and Lv-SPHK1-siRNA \#2; Fig. 4A) or treated with the SPHK1 inhibitor 5C (10 or $20 \mu \mathrm{M}$; Fig. 4B) for $24 \mathrm{~h}$; subsequently, a Transwell assay was used to determine the migratory ability. The results indicated that the migration ability was markedly lower in the Lv-SPHK1-siRNA and SPHK1 inhibitor groups compared with that in the control group (Fig. 4A and B). A similar trend was also observed regarding the invasion ability of TC cells (Fig. 4C and D).

Intrasplenic injection was then performed as a valid method (18) for assessing the metastatic ability of TPC-1 cells in vivo. Injection of control, Lv-NC-siRNA or
Table I. Association between p-SPHK1 and clinicopathological factors.

\begin{tabular}{|c|c|c|c|}
\hline \multirow[b]{2}{*}{ Factor } & \multicolumn{2}{|c|}{ p-SPHK1 $(n=53)$} & \multirow[b]{2}{*}{ P-value } \\
\hline & $\begin{array}{c}\text { Negative } \\
(\mathrm{n}, \%)\end{array}$ & $\begin{array}{c}\text { Positive } \\
(\mathrm{n}, \%)\end{array}$ & \\
\hline Age (years) & & & 0.591 \\
\hline$>45$ & $13(25)$ & $8(15)$ & \\
\hline$\leq 45$ & $18(34)$ & $14(26)$ & \\
\hline Gender & & & 0.733 \\
\hline Male & $17(32)$ & $14(26)$ & \\
\hline Female & $14(26)$ & $8(15)$ & \\
\hline Primary tumor ${ }^{\mathrm{a}}$ & & & 0.021 \\
\hline $\mathrm{T} 1$ & $14(26)$ & $6(12)$ & \\
\hline $\mathrm{T} 2, \mathrm{~T} 3, \mathrm{~T} 4$ & $17(32)$ & $16(30)$ & \\
\hline TNM stage & & & 0.008 \\
\hline I and II & $19(36)$ & $9(13)$ & \\
\hline III and IV & $12(26)$ & $13(25)$ & \\
\hline $\mathrm{N}$ classification & & & 0.841 \\
\hline N0 & $13(25)$ & $8(15)$ & \\
\hline N1 & $18(34)$ & $12(26)$ & \\
\hline
\end{tabular}

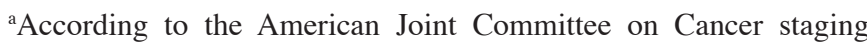
system. TNM, tumor-nodes-metastasis; p-SPHK1, phosphorylated sphingosine kinase $1 ; \mathrm{N}$, nodes

Lv-SPHK1-siRNA\#1 cells into the spleen of NOD/SCID mice was performed and mice in the control and Lv-NC-siRNA\#1 groups developed metastatic tumors after 6 weeks. Tumors were detected in the liver $(5 / 5$ in the control and Lv-NC-siRNA group), colon (3/5 in the control and 4/5 in the Lv-NC-siRNA group), lung (4/5 in the control and $3 / 5$ in the Lv-NC-siRNA group), spleen (3/5 in the control group and $2 / 5$ in the Lv-NC-siRNA group) and lymph nodes (4/5 in the control group and Lv-NC-siRNA group). By contrast, the mice in the Lv-SPHK1-siRNA\#1 group exhibited significantly fewer metastatic nodules in these organs $(\mathrm{P}<0.05$; Table II). Taken together, these results demonstrated that the metastatic capacity of TC cells was positively correlated with SPHK1 expression.

Inhibition of SPHK1 expression sensitizes TC cells to cisplatin and doxorubicin. The present study investigated the effects of silencing SPHK1 expression on the viability of TC cell lines. TPC-1 and WRO cells were selected for further study. TPC-1 and WRO cells were transfected with Lv-NC-siRNA or LV-SPHK1-siRNA\#1 for $24 \mathrm{~h}$, followed by treatment with $10 \mu \mathrm{M}$ cisplatin (Fig. 5A) or $10 \mu \mathrm{M}$ doxorubicin (Fig. 5B) for 48 h. The CCK-8 assay demonstrated that the cell viability in the Lv-SPHK1-siRNA\#1 group was obviously decreased compared with that in the control or LV-NC-siRNA group (Fig. 5A and B). A similar result was also obtained in TPC-1 and WRO cells treated with SPHK1 inhibitor 5C (Fig. 5C and D). In brief, these results suggested that induction of antiproliferative effects is a major mechanism by which silencing 
A

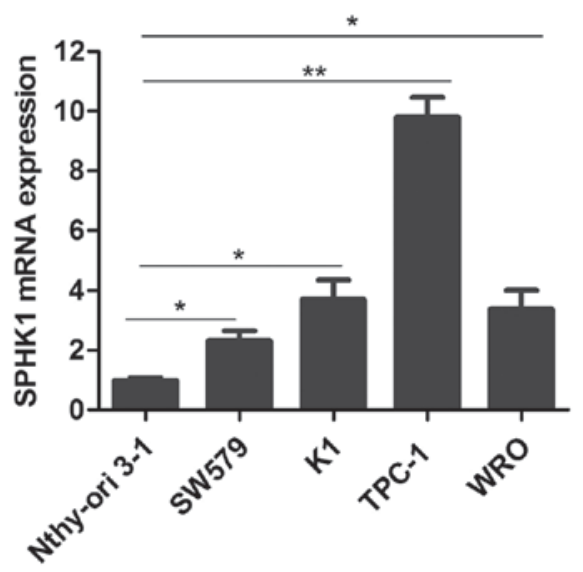

C

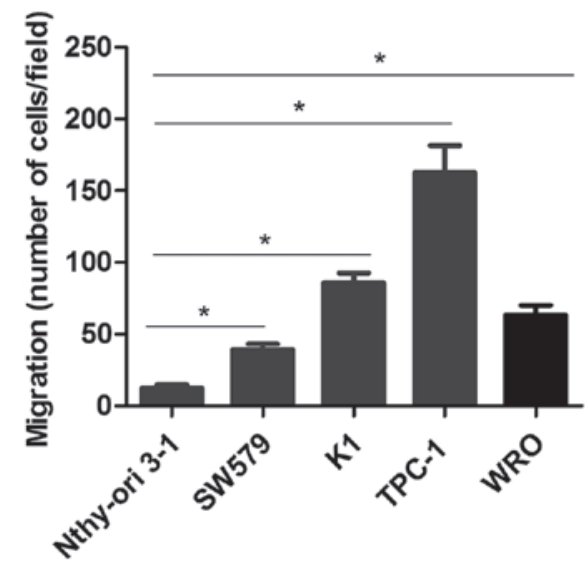

E

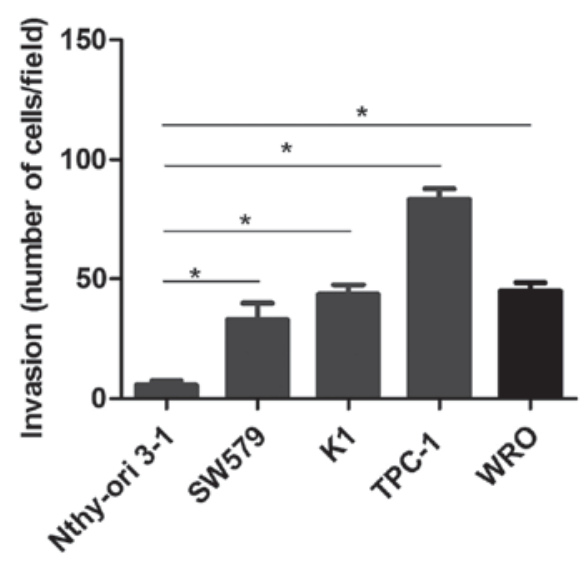

B

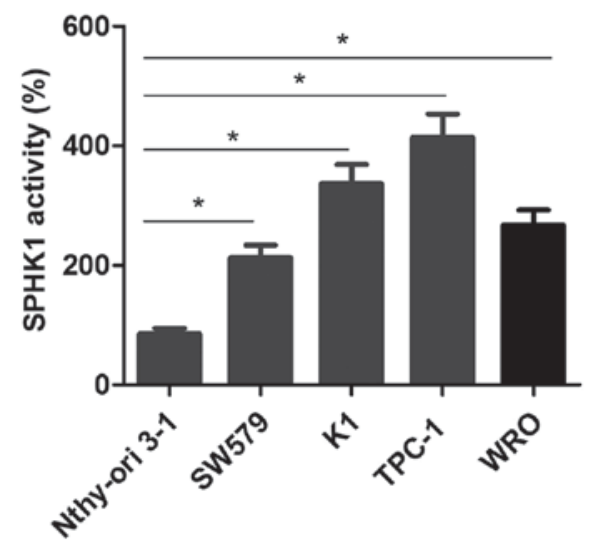

D

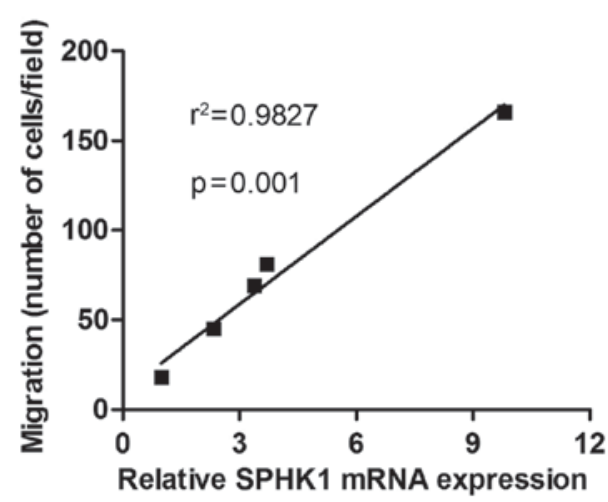

$\mathbf{F}$

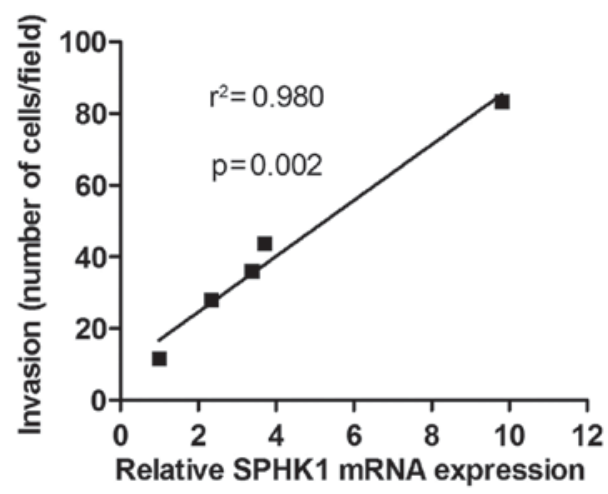

Figure 3. SPHK1 levels in TC cell lines are higher compared with those in normal thyroid cells and the metastatic ability of TC cells is positively associated with SPHK1 expression. (A) SPHK1 mRNA expression was detected in TC cell lines and the normal thyroid cell line Nthy-ori 3-1. (B) The activity of SPHK1 was determined in TC cell lines and a normal thyroid cell line. (C) The migration ability was determined in the TC cell lines SW579, K1, TPC-1 and WRO, as well as the normal thyroid cell line Nthy-ori 3-1 as a control. (D) Pearson's correlation analysis was used to determine the correlation between SPHK1 expression and migration ability of TC cells $\left(\mathrm{r}^{2}=0.9827, \mathrm{P}=0.001\right)$. (E) The invasion ability was determined in the TC cell lines SW579, K1, TPC-1 and WRO, and the normal thyroid cell line Nthy-ori 3-1 as a control. (F) Pearson's correlation analysis was used to determine the correlation between SPHK1 expression and invasion ability of TC cells $\left(\mathrm{r}^{2}=0.980, \mathrm{P}=0.002\right)$. Values are expressed as the mean \pm standard deviation. ${ }^{*} \mathrm{P}<0.05 ;{ }^{* * *} \mathrm{P}<0.01$. SPHK1, sphingosine kinase 1 ; TC, thyroid carcinoma.

of SPHK1 expression modulates the sensitivity of TC cells to cisplatin and doxorubicin.
SPHK1 increases SIP levels and activates the Notch signaling pathway via SIP receptor 3 (S1PR3). It has been 
Table II. Metastatic capacity of SPHK1 cells in vivo.

\begin{tabular}{|c|c|c|c|c|c|c|}
\hline Group & Liver & Colon & Lung & Spleen & Lymph nodes & P-value \\
\hline Control & $5 / 5$ & 3.5 & $4 / 5$ & $3 / 5$ & $4 / 5$ & \\
\hline Lv-NC-siRNA & $5 / 5$ & $4 / 5$ & $3 / 5$ & $2 / 5$ & $4 / 5$ & 0.021 \\
\hline 1V-SPHK1-siRNA\#1 & $2 / 5$ & $1 / 5$ & $1 / 5$ & $0 / 5$ & $1 / 5$ & \\
\hline
\end{tabular}

At 6 weeks following intrasplenic injection, the tumors of various organs were examined. SPHK1, sphingosine kinase; Lv, lentiviral vector; $\mathrm{NC}$, negative control; siRNA, small interfering RNA.

A

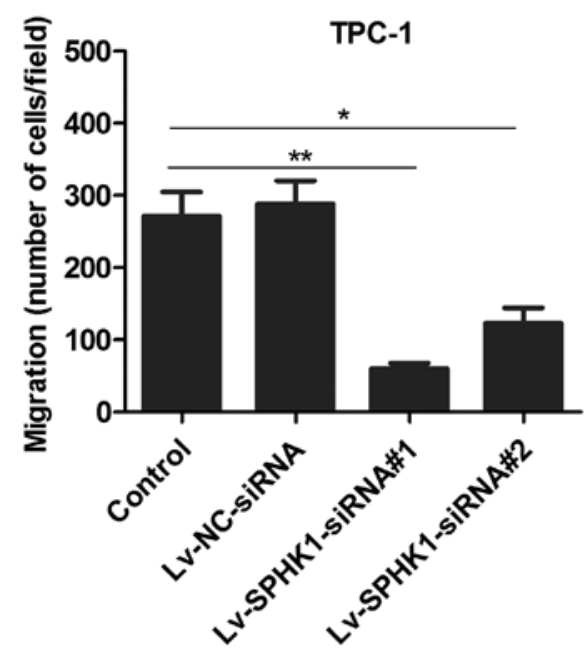

C

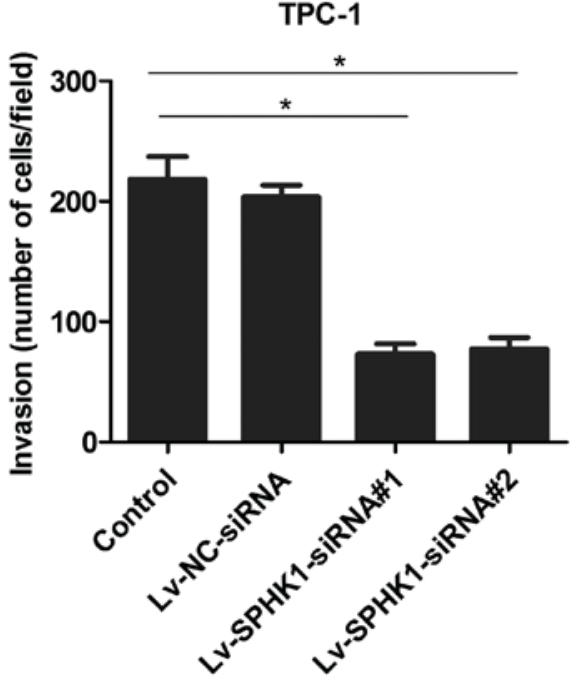

B

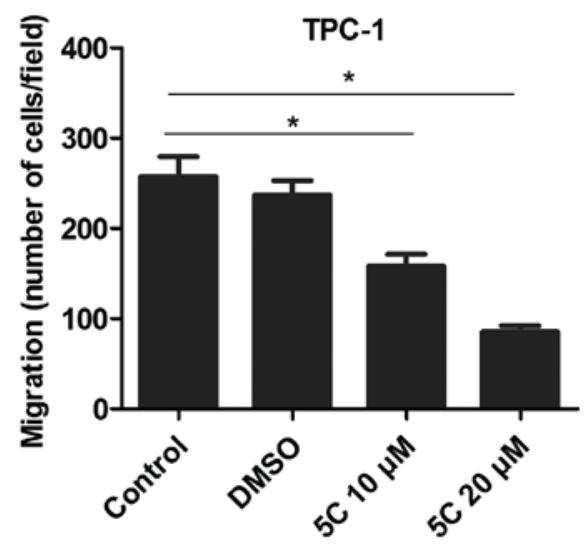

D

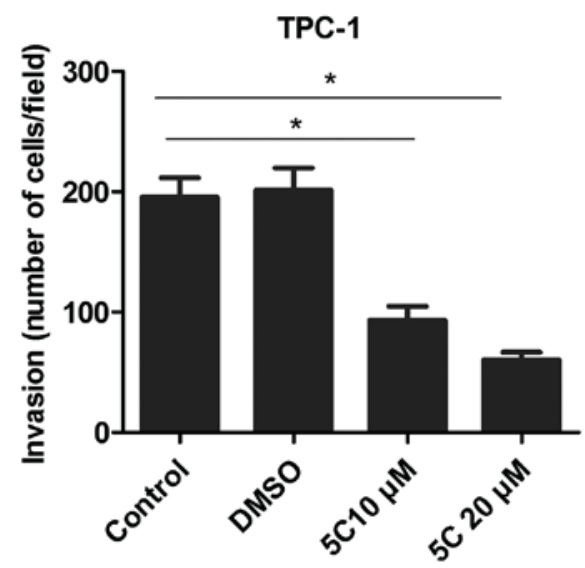

Figure 4. SPHK1 knockdown impairs the migration and invasion ability of TC cells in vitro, and reduces the metastatic ability of TC cells in vivo. (A) TPC-1 cells were transfected with Lv-NC-siRNA, Lv-SPHK1-siRNA\#1 or Lv-SPHK1-siRNA\#2 at a multiplicity of infection of 20. The migration ability was determined by a Transwell assay. (B) TPC-1 cells were treated with SPHK1 inhibitor 5C (10 or $20 \mu \mathrm{M})$ for $24 \mathrm{~h}$ and the migration ability was determined as in A. (C) TPC-1 cells were treated as in A and the invasion ability was detected by a Transwell assay. (D) TPC-1 cells were treated as in B the invasion ability was determined as in C. Values are expressed as the mean \pm standard deviation. ${ }^{*} \mathrm{P}<0.05 ;{ }^{* *} \mathrm{P}<0.01$.

reported that S1PR has an important role in the regulation of cell survival by SPHK1 (15). Thus, the present study mainly focused on Wnt, Hedgehog and Notch signaling as candidate downstream pathways of S1PR. The present results indicated that Hes1 was downregulated after TPC-1 cells were treated with Lv-SPHK1-siRNA\#1 (Fig. 6A) or SPHK1 inhibitor 
A

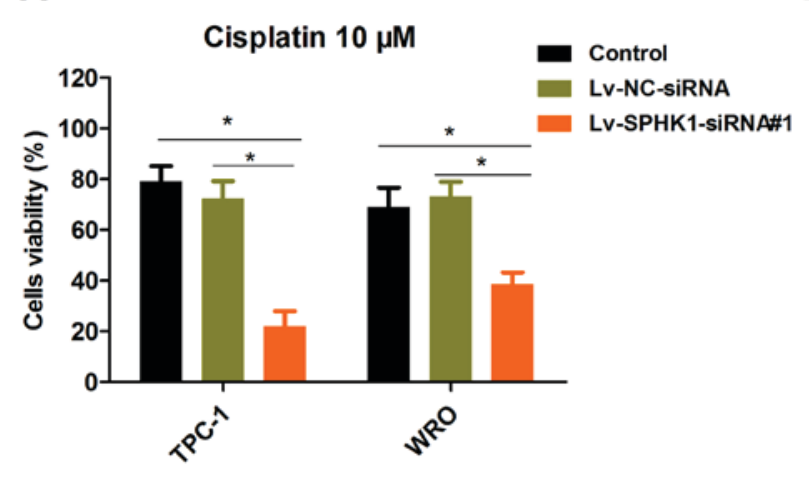

C

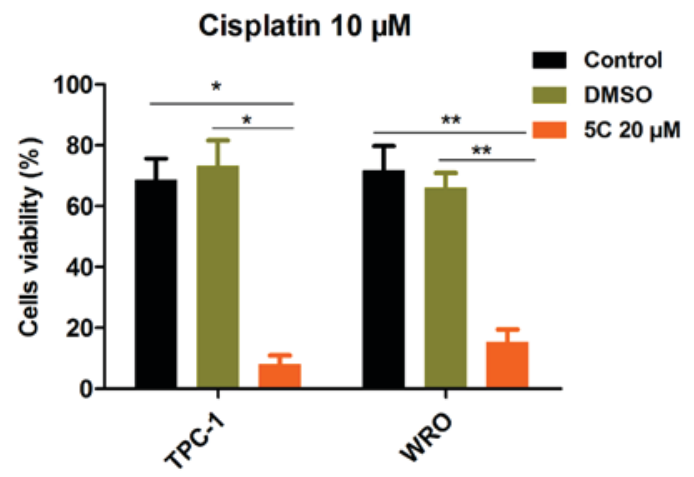

B

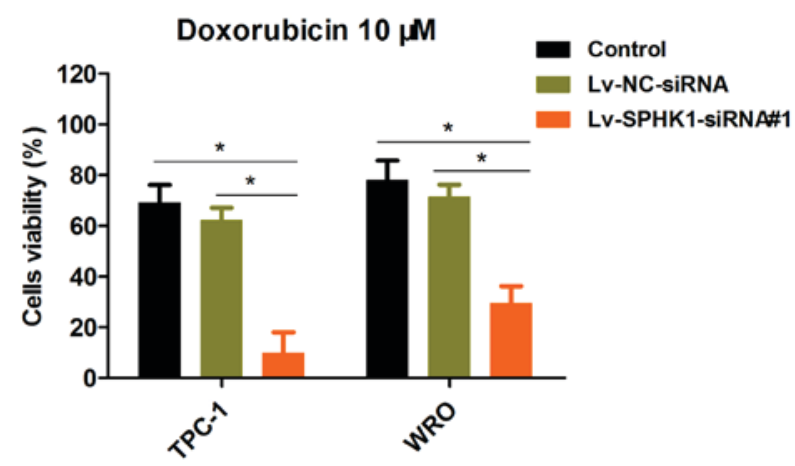

D

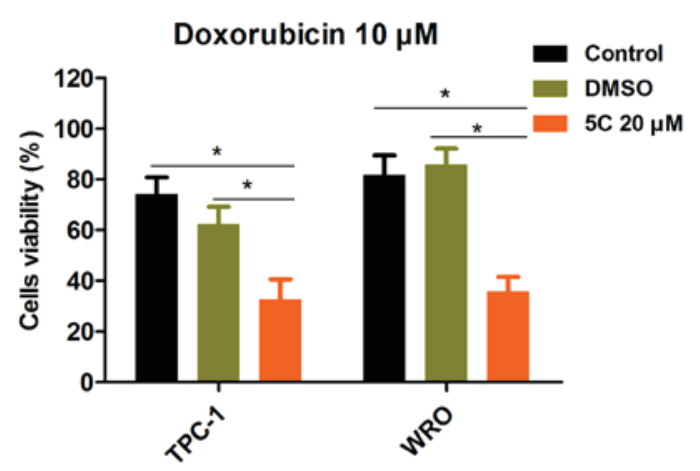

Figure 5. Silencing of SPHK1 expression sensitizes TC cells to cisplatin and doxorubicin. (A and B) Viability of TC cells transfected with Lv-NC-siRNA or Lv-SPHK1-siRNA\#1 at a multiplicity of infection of $20 \mathrm{for} 24 \mathrm{~h}$, followed by treatment with (A) $10 \mu \mathrm{M}$ cisplatin or (B) $10 \mu \mathrm{M}$ doxorubicin for $48 \mathrm{~h}$. (C and D) Viability of TC cells treated with DMSO or $20 \mu \mathrm{M} 5 \mathrm{C}$ for $24 \mathrm{~h}$, followed by treatment with (C) $10 \mu \mathrm{M}$ cisplatin or (D) $10 \mu \mathrm{M}$ doxorubicin for $48 \mathrm{~h}$. A Cell Counting Kit- 8 assay was used to assess cell viability. Values are expressed as the mean \pm standard deviation. " P $<0.05$; * ${ }^{* *}<0.01$. Lv-SPHK1-siRNA, lentiviral vector expressing small interfering RNA targeted to SPHK1; NC, negative control; TC, thyroid carcinoma; DMSO, dimethyl sulfoxide.

5C (Fig. 6B) for $24 \mathrm{~h}$. These results suggest that the Notch signaling pathway was repressed by silencing of SPHK1 expression. By contrast, stimulation with S1P obviously upregulated the expression of Hes1 in TPC-1 cells rather than LPA (Fig. 6C). Furthermore, the protein expression of Hes1 was also downregulated in TPC-1 cells transfected with Lv-SPHK1-siRNA\#1 (Fig. 6D). However, DKK1 and Gli1 were not significantly affected (Fig. 6A and B). In addition, the induction of Hes1 expression by S1P was abrogated by S1PR3 antagonists TY52156 $(2 \mu \mathrm{M})$ and CAY10444 $(5 \mu \mathrm{M})$, but not by S1PR2 antagonist JTE013 $(2 \mu \mathrm{M})$ (Fig. 6E). These results indicated that there was a reduction, but not an abrogation as for the other antagonists. To further investigate whether S1P activates the Notch signaling pathway, the cleavage of Notch in TPC-1 cells was also detected. The expression of N1ICD was significantly upregulated in TPC-1 cells treated with S1P, while overexpression of N1ICD was performed as a positive control (Fig. 6F). Collectively, these results suggested that SPHK1 induces the activation of the Notch signaling pathway and that SPHK1 mediates S1P-induced Notch signaling pathway activation via S1PR3.

\section{Discussion}

In contrast to the anti-proliferative effects of ceramide and sphingosine, S1P regulates cell proliferation, migration and survival, promotes angiogenesis and has a pivotal role in the pathogenesis of inflammation as well as chemotaxis of lymphocytes (26). As a pivotal enzyme for S1P production in vivo, SPHK1 activated by extracellular receptor agonist mainly has a role in regulating extracellular S1P levels $(27,28)$. In addition, numerous studies have indicated that inhibition of SPHK1 impairs tumor cell proliferation (29,30). Zhang et al (31) have reported that inhibition of SPHK1 expression significantly decreased the migration capability of hepatocellular carcinoma cells. The present study indicated that SPHK1 expression was markedly upregulated in TC compared with those in normal thyroid tissues and was closely associated with a poorer OS and PFS in patients with TC. These results were consistent with those of previous studies and imply that SPHK1 may be closely associated with the occurrence, progression and development of TC.

Activation of SPHK1 is a crucial factor for cell proliferation, transformation, metastasis and chemoresistance (23). It was reported to be upregulated in multiple types of solid tumor and to be closely correlated with poor prognosis (17). The observations of the present study were in line with the above in patients with TC who poorly responded to doxorubicin treatment (3). Furthermore, the present results indicated that the cytotoxic effects of chemotherapeutic drugs on TC cells were promoted by siRNA-mediated knockdown or pharmacological inhibition of SPHK1 in vitro. A previous study indicated that inhibition of SPHK1 activity by treatment with safingol markedly potentiated epigallocatechin gallate-induced apoptotic 
A

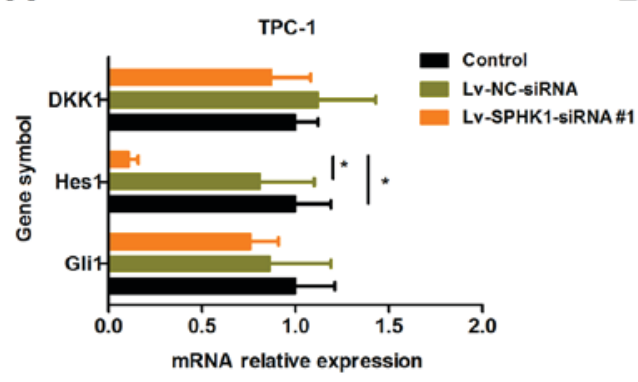

D

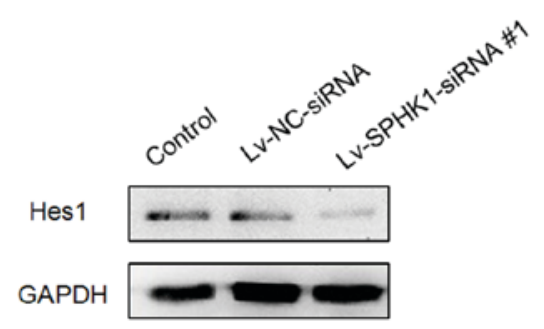

B

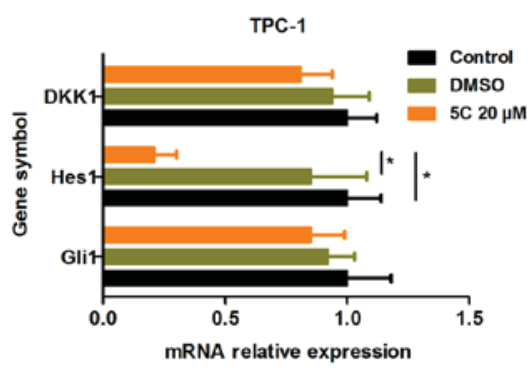

E

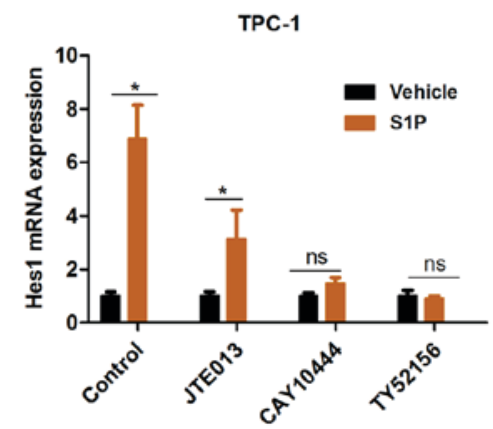

C

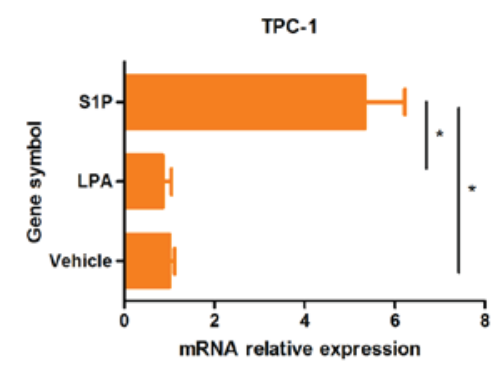

F

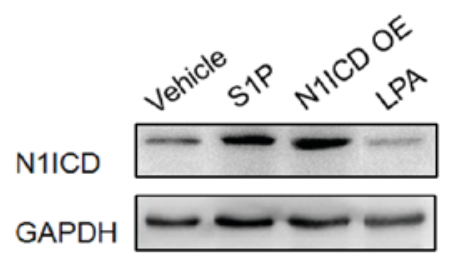

Figure 6. SPHK1 causes an increase in S1P levels and activates the Notch signaling pathway via S1PR3. (A and B) TPC-1 cells were (A) transfected with Lv-NC-siRNA or Lv-SPHK1-siRNA\#1 at an MOI of 20 for $24 \mathrm{~h}$, or (B) treated with DMSO or $20 \mu \mathrm{M} 5 \mathrm{C}$ for $24 \mathrm{~h}$, and the expression of DKK1, Hes1 and Gli1 was determined by an RT-qPCR assay. (C) TPC-1 cells were treated with S1P (300 nM) and LPA (300 nM) for 24 h, and the expression of Hes1 was determined by RT-qPCR. (D) TPC-1 cells were transfected with Lv-NC-siRNA or Lv-SPHK1-siRNA\#1 at an MOI of 20 for 72 h, and western blot analysis was used to determine the protein expression of Hes1. (E) Effects of JTE013 ( $2 \mu \mathrm{M})$, CAY10444 (5 $\mu$ M) or TY52156 (2 $\mu$ M) on S1P-induced Hes1 expression. Values are expressed as the mean \pm standard deviation. * $\mathrm{P}<0.05$. (F) Effects of S1P, LPA or N1ICD OE on N1ICD expression; GAPDH was used as a control. N1ICD OE, Notch1 intracellular domain overexpression vector; Lv-SPHK1-siRNA, lentiviral vector expressing small interfering RNA targeted to SPHK1; ns, no significance; NC, negative control; TC, thyroid carcinoma; MOI, multiplicity of infection; DMSO, dimethyl sulfoxide; DKK1, dickkopf 1; Hes1, hairy and enhancer of split-1; RT-qPCR, reverse transcription-quantitative polymerase chain reaction; S1P, sphingosine-1-phosphate; LPA, lysophosphatidic acid.

activity in vitro and in vivo (32). The results of the present study were consistent with several studies on other cancer types (33-35). In addition, inhibition of SPHK1 was demonstrated to sensitize TC cells to chemotherapeutic drugs, including 5-fluorouracil, docetaxel, doxorubicin and cisplatin. Taken together, SPHK1 inhibitors, alone or in combination with conventional anti-cancer drugs, have been demonstrated to have marked anti-tumor effects.

Furthermore, the results of the present study not only demonstrated that SPHK1 expression was markedly upregulated in TC tissues and cell lines, but also indicated that the upregulation of SPHK1 has a significant role in the invasion and metastasis of TC cells. A previous study indicated that SPHK1-5C (as utilized in the present study), a chemotherapeutic drug, significantly inhibited the serum-induced growth and survival of TC cells via the extracellular signal-regulated kinase/AKT pathway (36). However, the downstream mechanisms of the activated SPHK1/S1P pathway that promote the invasion and migration of TC cells have remained to be elucidated. The results of the present study indicated that the Notch target gene Hes1 was evidently upregulated in TPC-1 cells following stimulation with S1P and S1P-induced Hes1 expression was abrogated by S1PR3 antagonists, which implied that activation of the SPHK1/S1P pathway may promote the invasion and migration of TC cells. Furthermore, it was demonstrated that inhibition of SPHK1 expression interferes with the S1P/Notch signaling pathway and that it impairs the migration and invasion of TC cells in vitro as well as the metastatic capacity of TC in an in vivo metastasis model. Accordingly, the development of SPHK1-targeted therapeutics to improve the prognosis of patients with TC should be further investigated.

\section{Acknowledgements}

TC tissue and normal adjacent samples were immunostained by the Department of Surgical Pathology, Southwest Hospital of the Third Military Medical University (Chongqing, China).

\section{Funding}

No funding was received.

\section{Availability of data and materials}

The analyzed data sets generated during the present study are available from the corresponding author on reasonable request.

\section{Authors' contributions}

$\mathrm{ZZ}$ analyzed and interpreted the patient data. ZZ and JM performed the experiments, including the immunohistological staining. $\mathrm{BH}$ was responsible for the patient sample collection. $\mathrm{ZZ}$ and $\mathrm{BH}$ drafted the manuscript. YZ performed the 
statistical analysis. SW designed this study. All authors read and approved the final manuscript.

\section{Ethics approval and consent to participate}

All patients provided informed consent and the present study was approved by the medical Ethics Committee of the Third Military Medical University (Chongqing, China). All procedures for animal experiments were approved by the Laboratory Animal Welfare and Ethics Committee of the Third Military Medical University (Chongqing, China; no. 20151117083) and were performed in accordance with institutional guidelines.

\section{Consent for publication}

Not applicable.

\section{Competing interests}

All authors have no conflict of interest to declare.

\section{References}

1. Schmidt D and Kuwert T: Hybrid molecular imaging in differentiated thyroid carcinoma. Front Horm Res 45: 37-45, 2016.

2. Grewal RK, Ho A and Schöder H: Novel approaches to thyroid cancer treatment and response assessment. Semin Nucl Med 46: 109-118, 2016.

3. Nixon IJ: Well-differentiated thyroid cancer-are you overtreating your patients? Endokrynol Pol 67: 60-66, 2016.

4. Frampton JE: Lenvatinib: A review in refractory thyroid cancer. Target Oncol 11: 115-122, 2016.

5. Williams D: Thyroid growth and cancer. Eur Thyroid J 4: 164-173, 2015.

6. Schmid D, Ricci C, Behrens G and Leitzmann MF: Adiposity and risk of thyroid cancer: A systematic review and meta-analysis. Obes Rev 16: 1042-1054, 2015.

7. Bae YJ, Schaab M and Kratzsch J: Calcitonin as biomarker for the medullary thyroid carcinoma. Recent Results Cancer Res 204: 117-137, 2015.

8. Patmanathan SN, Wang W, Yap LF, Herr DR and Paterson IC: Mechanisms of sphingosine 1-phosphate receptor signalling in cancer. Cell Signal 34: 66-75, 2017.

9. Farez MF and Correale J: Sphingosine 1-phosphate signaling in astrocytes: Implications for progressive multiple sclerosis. J Neurol Sci 361: 60-65, 2016.

10. Guerrero M, Urbano M and Roberts E: Sphingosine 1-phosphate receptor 1 agonists: A patent review (2013-2015). Expert Opin Ther Pat 26: 455-470, 2016.

11. Kwong E, Li Y, Hylemon PB and Zhou H: Bile acids and sphingosine-1-phosphate receptor 2 in hepatic lipid metabolism. Acta Pharm Sin B 5: 151-157, 2015.

12. Sanllehí P, Abad JL, Casas J and Delgado A: Inhibitors of sphingosine-1-phosphate metabolism (sphingosine kinases and sphingosine-1-phosphate lyase). Chem Phys Lipids 197: 69-81, 2016.

13. Furuya H, Shimizu Y, Tamashiro PM, Iino K, Bielawski J, Chan OTM, Pagano I and Kawamori T: Sphingosine kinase 1 expression enhances colon tumor growth. J Transl Med 15: 120, 2017.

14. Huang LS and Natarajan V: Sphingolipids in pulmonary fibrosis. Adv Biol Regul 57: 55-63, 2015.

15. Lu PH, Chen MB, Liu YY, Wu MH, Li WT, Wei MX, Liu CY and Qin SK: Identification of sphingosine kinase 1 (SphK1) as a primary target of icaritin in hepatocellular carcinoma cells. Oncotarget 8: 22800-22810, 2017.

16. Li S, Zhou Y, Zheng X, Wu X, Liang Y, Wang S and Zhang Y: Sphk1 promotes breast epithelial cell proliferation via NF- $\mathrm{B}$-p65-mediated cyclin D1 expression. Oncotarget 7: 80579-80585, 2016.

17. Fan Z, Jiang H, Wang Z and Qu J: Atorvastatin partially inhibits the epithelial-mesenchymal transition in A549 cells induced by TGF- $\beta 1$ by attenuating the upregulation of SphK1. Oncol Rep 36: 1016-1022, 2016
18. Liang W, Xie Z, Cui W, Guo Y, Xu L, Wu J and Guan H: Comprehensive gene and microRNA expression profiling reveals a role for miRNAs in the oncogenic roles of SphK1 in papillary thyroid cancer. J Cancer Res Clin Oncol 143: 601-611, 2017.

19. Guan H, Liu L, Cai J, Liu J, Ye C, Li M and Li Y: Sphingosine kinase 1 is overexpressed and promotes proliferation in human thyroid cancer. Mol Endocrinol 25: 1858-1866, 2011.

20. Murakami M, Ichihara M, Sobue S, Kikuchi R, Ito H, Kimura A, Iwasaki T, Takagi A, Kojima T, Takahashi M, et al: RET signaling-induced SPHK1 gene expression plays a role in both GDNF-induced differentiation and MEN2-type oncogenesis. J Neurochem 102: 1585-1594, 2007.

21. Brünnert D, Sztachelska M,Bornkessel F, Treder N, WolczynskiS, Goyal P and Zygmunt M: Lysophosphatidic acid and sphingosine 1-phosphate metabolic pathways and their receptors are differentially regulated during decidualization of human endometrial stromal cells. Mol Hum Reprod 20: 1016-1025, 2014.

22. Datta A, Loo SY, Huang B, Wong L, Tan SS, Tan TZ, Lee SC, Thiery JP, Lim YC, Yong WP, et al: SPHK1 regulates proliferation and survival responses in triple-negative breast cancer. Oncotarget 5: 5920-5933, 2014.

23. Ribeiro FR, Meireles AM, Rocha AS and Teixeira MR: Conventional and molecular cytogenetics of human non-medullary thyroid carcinoma: Characterization of eight cell line models and review of the literature on clinical samples. BMC Cancer 8: $371,2008$.

24. Nakamura T, Matsuyama N, Kirino M, Kasai M, Kiyohara S and Ikenaga T: Distribution, innervation, and cellular organization of taste buds in the sea catfish, plotosus japonicus. Brain Behav Evol 89: 209-218, 2017.

25. Livak KJ and Schmittgen TD: Analysis of relative gene expression data using real-time quantitative PCR and the 2(-Delta Delta C(T)) method. Methods 25: 402-408, 2001.

26. Panneer Selvam S, De Palma RM, Oaks JJ, Oleinik N Peterson YK, Stahelin RV, Skordalakes E, Ponnusamy S, Garrett-Mayer E, Smith CD and Ogretmen B: Binding of the sphingolipid S1P to hTERT stabilizes telomerase at the nuclear periphery by allosterically mimicking protein phosphorylation. Sci Signal 8: ra58, 2015.

27. Liu H, Ma Y, He HW, Zhao WL and Shao RG: SPHK1 (sphingosine kinase 1) induces epithelial-mesenchymal transition by promoting the autophagy-linked lysosomal degradation of CDH1/E-cadherin in hepatoma cells. Autophagy 13: 900-913, 2017.

28. Sukocheva O, Wadham C, Gamble J and Xia P: Sphingosine-1-phosphate receptor 1 transmits estrogens' effects in endothelial cells. Steroids 104: 237-245, 2015.

29. Xu Y, Dong B, Huang J, Kong W, Xue W, Zhu Y, Zhang J and Huang Y: Sphingosine kinase 1 is overexpressed and promotes adrenocortical carcinoma progression. Oncotarget 7: 3233-3244, 2016.

30. Okada T, Ding G, Sonoda H, Kajimoto T, Haga Y, Khosrowbeygi A, Gao S, Miwa N, Jahangeer S and Nakamura S: Involvement of $\mathrm{N}$-terminal-extended form of sphingosine kinase 2 in serum-dependent regulation of cell proliferation and apoptosis. J Biol Chem 280: 36318-36325, 2005.

31. Zhang Z, Yan Z, Yuan Z, Sun Y, He H and Mai C: SPHK1 inhibitor suppresses cell proliferation and invasion associated with the inhibition of NF- $\mathrm{B}$ p pathway in hepatocellular carcinoma. Tumour Biol 36: 1503-1509, 2015.

32. Tsukamoto S, Huang Y, Kumazoe M, Lesnick C, Yamada S, Ueda N, Suzuki T, Yamashita S, Kim YH, Fujimura Y, et al: Sphingosine kinase-1 protects multiple myeloma from apoptosis driven by cancer-specific inhibition of RTKs. Mol Cancer Ther 14: 2303-2312, 2015.

33. Yu H, Duan P, Zhu H and Rao D: miR-613 inhibits bladder cancer proliferation and migration through targeting SphK1. Am J Transl Res 9: 1213-1221, 2017.

34. Zhang S, Deng Z, Yao C, Huang P, Zhang Y, Cao S and Li X: AT7867 inhibits human colorectal cancer cells via AKT-Dependent and AKT-Independent mechanisms. PLoS One 12: e0169585, 2017.

35. Li W, Li J, Wang Y, Zhang K, Li N, Tian Z, Ni B, Wang H and Ruan Z: Sphingosine kinase 1 is a potential therapeutic target for nasopharyngeal carcinoma. Oncotarget 7: 80586-80598, 2016.

36. Zhu P, Liao LY, Zhao TT, Mo XM, Chen GG and Liu ZM: GPER/ERK\&;AKT/NF- $\kappa$ B pathway is involved in cadmium-induced proliferation, invasion and migration of GPER-positive thyroid cancer cells. Mol Cell Endocrinol 442: 68-80, 2017. 